University of New England DUNE: DigitalUNE

9-19-2016

\title{
Positive Influence Of A Dental Anaesthesia Simulation Model On The Perception Of Learning By Mexican Dental Students
}

\author{
C. López-Cabrera
}

Universidad Veracruzana

E. J. Hernández-Rivas

Universidad Veracruzana

Takashi Komabayashi

Clinical Professor, University of New England College of Dental Medicine, tkomabayashi@une.edu

E. L. Galindo-Reyes

Universidad Veracruzana

D. Tallabs-López

MORE Center of Organizational and Personal Development

See next page for additional authors

Follow this and additional works at: http://dune.une.edu/cdm_facpubs

Part of the Dentistry Commons, Educational Methods Commons, and the Higher Education $\underline{\text { Commons }}$

\section{Recommended Citation}

López-Cabrera, C.; Hernández-Rivas, E. J.; Komabayashi, Takashi; Galindo-Reyes, E. L.; Tallabs-López, D.; and Cerda-Cristerna, Bernardino Isaac, "Positive Influence Of A Dental Anaesthesia Simulation Model On The Perception Of Learning By Mexican Dental Students" (2016). Dental Medicine Faculty Publications. Paper 7.

http://dune.une.edu/cdm_facpubs/7

This Article is brought to you for free and open access by the College of Dental Medicine at DUNE: DigitalUNE. It has been accepted for inclusion in Dental Medicine Faculty Publications by an authorized administrator of DUNE: DigitalUNE. For more information, please contact

bkenyon@une.edu. 
Authors

C. López-Cabrera, E. J. Hernández-Rivas, Takashi Komabayashi, E. L. Galindo-Reyes, D. Tallabs-López, and Bernardino Isaac Cerda-Cristerna 


\section{Positive influence of a dental anesthesia simulation model on the perception of learning by Mexican dental students}

Authors: C. López-Cabrera, E. J. Hernández-Rivas, T. Komabayashi, E. L. Galindo-

Reyes, D. Tallabs-López, B. I. Cerda-Cristerna

\section{ABSTRACT}

Introduction: This study evaluated the influence of 3-repetition training with a dental anesthesia simulation model (DASM) on the perception of learning by dental students.

Materials and methods: Dental students who had never used a dental anesthesia technique were randomly divided into 2 groups that were taught the anterior superior alveolar nerve infiltrative anesthesia technique. Group $1(\mathrm{G} 1, \mathrm{~N}=10)$ followed a 3-stage learning method: $1^{\text {st }}$ ) theoretical lecture, $2^{\text {nd }}$ ) clinical demonstration, and $3^{\text {rd }}$ ) DASM training, including 3 repetitions of the anesthesia technique. Group $2(\mathrm{G} 2, \mathrm{~N}=10)$ followed only the $1^{\text {st }}$ and $2^{\text {nd }}$ stages. The students in both groups then performed the anesthesia technique. The perception of the students was evaluated by 4 learning concepts. Each was evaluated with a 5-point Likert Scale questionnaire. The average score of each item of the questionnaire for G1 was compared with that of G2. Statistically significant differences were identified with the Mann-Whitney test. The average working time of each group was timed and compared by Student's $t$-test to identify possible statistically significant differences.

Results: Students in G1 showed higher average scores of perception in controlling the handling of the dental syringe and confidence in performing the injection $(p<0.05)$, and showed an average working time shorter than that of the students in $\mathrm{G} 2 \quad(p<0.05)$. Conclusion: The DASM positively influenced the perception learning of the dental students; it increased their confidence and syringe handling ability, as well as skills to perform the injection of anesthesia more quickly.

Key words: dental anesthesia, dental education, phantom head, simulation, training model. 


\section{INTRODUCTION}

Simulation model learning strategies have been applied in dental education for more than 100 years (1). This learning system helps to develop students' psychomotor skills before they perform a clinical intervention for the first time, to teach or improve students' clinical manipulation skills, and to increase safety for patients being treated by inexperienced students or young clinicians. With this system, a student might practice a clinical procedure several times without performing an intervention on a patient $(1,2)$. Simulation models have significantly influenced learning in several dental areas, including endodontics, periodontics, dental hygiene, and operative dentistry $(1,3,4)$.

Simulation models have received little attention in the field of dental anesthesia, even if those tools have been proven for the teaching of local anesthesia techniques in other biomedical areas (5-7); indeed, only a few dental schools have included dental anesthesia simulation models (DASM) in their curricula (8). The student-to-student administration model-in which a student administers an anesthetic injection to another student-is still the most common method for initial dental anesthesia practice; however, this method invokes ethical issues and causes anxiety for practitioners $(9,10)$. Dental students consider a simulation model to be useful practice before they inject a patient for the first time, and they have expressed agreement with the helpfulness of DASM after using it $(9,11,12)$. However, some studies have reported that the benefit of DASM is unclear from a clinical

point of view (13-15). Dental students who followed a learning protocol with DASM showed better theoretical knowledge on a knowledge acquisition assessment test (KAAT) than did students who did not follow the protocol, but when the KAAT evaluated clinical knowledge, the 2 groups obtained similar mean scores with no statistically significant differences (14). In addition, students reported being no more confident in their first-time administration of local anesthesia even after receiving previous training with a simulation model (13). Conversely, students trained with a DASM had a generally higher positive perception in terms of preparation and confidence when first administering local anesthesia; this perception was evaluated with a post-injection, 6-item questionnaire that was also given to untrained students (15). However, on only 1 of the items did those in the DASM- 
trained group show a mean score with a statistically significant difference when compared with the mean score of those in the untrained group (15).

For the benefits of DASM to be obtained, training protocols should first be explored to test its efficacy, and these protocols should be clearly described for best application. A major concern is that some studies have not detailed the training protocol; for instance, information such as the topics learned and the number of simulated injections has not been reported (13-15). In addition, studies have used simulators that produce an audible alarm if the dental needle comes into contact with a metal detector located at the anatomical puncture site, but these simulators are unsuitable for students practicing injections with an anesthetic solution, because the solution would damage the electrical system. Thus, from our point of view, the training might not involve a realistic, complete simulation of the anesthesia injection process.

In this study, we explored a learning protocol to overcome the concerns described above. Moreover, to the best of our knowledge, no reports exist on the use of DASM in Mexican dental schools, and our faculty has recently acquired a DASM to be implemented in the dental curriculum. Accordingly, we aimed to evaluate the impact of a DASM composed of a simulation model fixed in a phantom head on the perception of 4 psychomotor skills among dental students performing a first-time administration of dental anesthesia.

\section{MATERIALS AND METHODS}

An ethics committee approved the study, and all participants signed the informed consent. Twenty healthy second-year students in the 5-year dental program of the Faculty of Dentistry of Río Blanco, University of Veracruz, participated. All participants had successfully completed the didactic/theoretical portion of a dental anesthesiology course, in which they learned theoretical topics on the physiology of pain as well as the pharmacology and instrumentation of dental anesthesia. They had never performed a dental anesthetic technique on a patient. All students included in the study reported no allergy to lidocaine, benzocaine, or any other compound in a dental anesthesia solution. The participants were randomly divided into 2 groups: Group 1 (G1) $(n=10)$ and Group 2 (G2) $(n=10)$. 
Three-stage learning protocol: I) theoretical lecture, II) clinical demonstration, and III) DASM training

G1 and G2 followed a learning protocol (Table 1), and all instruction was provided by the professor of the dental anesthesia course (the corresponding author). Students in G1 followed the first, second, and third stages (Table 1), while those in G2 followed the first and second stages. The protocol was designed for students learning the infiltrative anesthesia technique for the anterior superior alveolar nerve (IASAN) with anesthesia on tooth 8 (right maxillary central incisor) and tooth 7 (right maxillary lateral incisor) by means of a technique based on Malamed's Handbook of Local Anesthesia (16). On the DASM stage, we used the typodont conduction anesthesia model (CAM, SUG2005-UL-SP, Nissin Dental Products, Nakagyo-ku, Kyoto, Japan) fixed in a phantom head (Nissin Dental Products) adapted to the back of a dental chair (Gnatus-México, Queretaro, Mexico) (Figure 1). When CAM was used, the electrical sensors were protected with silicone to avoid damage from the anesthetic solution; other electrical parts were protected with a disposable dental bib. The preclinical training in the DASM stage was performed with an aspirating syringe (M.A. Arain \& Brothers, Sialkot, Pakistan), an anesthetic cartridge (2\% lidocaine and 1:100,000 epinephrine, Zeyco, Guadalajara, Jalisco, Mexico), and a dental needle ( $25 \mathrm{~mm}$, gauge 27; Septodont, France). These were new for every repetition of the injection (3 repetitions).

\section{Clinical practice}

Twenty-four hours after the G1 DASM stage and 24 hours after the G2 theoretical lecture, both groups performed the IASAN in a student-to-student method (operator-to-receptor). The G1 students performed the IASAN on the G2 students, with the IASAN performed first. Three hours after finishing this stage, G2 students performed the IASAN on G1 students. The clinical practice was supervised by the professor in a clinical cubicle equipped with one dental chair. Before the anesthesia was administered, the sensitivity of each receptor's teeth 7 and 8 was tested with a cold solution (Hygenic Endo-Ice, ColtèneWhaledent, Cuyahoga Falls, OH, USA) 5 minutes after IASAN (17). The IASAN was considered a success if the receptor reported no sensitivity on the teeth in question. The 
Table 1. Three-stage learning protocol in group 1 and group 2

\begin{tabular}{|c|c|c|c|}
\hline Stage & Topics & G1 & G2 \\
\hline $\begin{array}{l}\text { First stage: } \\
\text { theoretical } \\
\text { lecture } \\
\text { (40 min) }\end{array}$ & $\begin{array}{l}\text { 1. Anatomical considerations of the anterior } \\
\text { superior alveolar nerve. } \\
\text { 2. Localization of the anatomical puncture site } \\
\text { - level of apical third tooth } 8 \text {-and drying of } \\
\text { the zone with a cotton tip. } \\
\text { 3. Application of topical anesthesia and } \\
\text { recommended contact time (two min). } \\
\text { 4. Assembly of the anesthesia cartridge and } \\
\text { dental needle on the aspirative dental } \\
\text { syringe. } \\
\text { 5. Performing injection and recommended } \\
\text { injection time (two min. per cartridge) } \\
\text { 6. Performing puncture and aspiration with the } \\
\text { dental syringe. } \\
\text { 7. Re-capping the dental needle with the one- } \\
\text { handed scoop technique }\end{array}$ & YES & YES \\
\hline $\begin{array}{l}\text { Second stage: } \\
\text { clinical } \\
\text { demonstration } \\
\text { by a clinical } \\
\text { professor } \\
\text { (30 min) }\end{array}$ & $\begin{array}{l}\text { 1. Anatomical considerations of the anterior } \\
\text { superior alveolar nerve. } \\
\text { 2. Localization of the anatomical puncture site } \\
\text { - level of apical third tooth } 8 \text {-and drying of } \\
\text { the zone with a cotton tip. } \\
\text { 3. Application of topical anesthesia and } \\
\text { recommended contact time (two min). } \\
\text { 4. Assembly of the anesthesia cartridge and } \\
\text { dental needle on the aspirative dental } \\
\text { syringe. } \\
\text { 5. Performing injection and recommended } \\
\text { injection time (two min. per cartridge) } \\
\text { 6. Performing puncture and aspiration with the } \\
\text { dental syringe. } \\
\text { 7. Re-capping the dental needle with the one- } \\
\text { handed scoop technique. }\end{array}$ & YES & YES \\
\hline $\begin{array}{c}\text { Third stage: } \\
\text { dental anesthesia } \\
\text { simulation training } \\
3 \text { repetitions } \\
\text { (total time, } 30 \mathrm{~min} \text { ) }\end{array}$ & $\begin{array}{l}\text { 1. Anatomical considerations of the anterior } \\
\text { superior alveolar nerve. } \\
\text { 2. Localization of the anatomical puncture site } \\
\text { - level of apical third tooth } 8 \text {-and drying of } \\
\text { the zone with a cotton tip. } \\
\text { 3. Application of topical anesthesia and } \\
\text { recommended contact time (two min). } \\
\text { 4. Assembly of the anesthesia cartridge and } \\
\text { dental needle on the aspirative dental } \\
\text { syringe. } \\
\text { 5. Performing injection and recommended } \\
\text { injection time (two min. per cartridge) } \\
\text { 6. Performing puncture and aspiration with the } \\
\text { dental syringe. } \\
\text { 7. Re-capping the dental needle with the one- } \\
\text { handed scoop technique. }\end{array}$ & YES & $\mathrm{NO}$ \\
\hline
\end{tabular}

\section{G1: group 1, G2: group 2.}



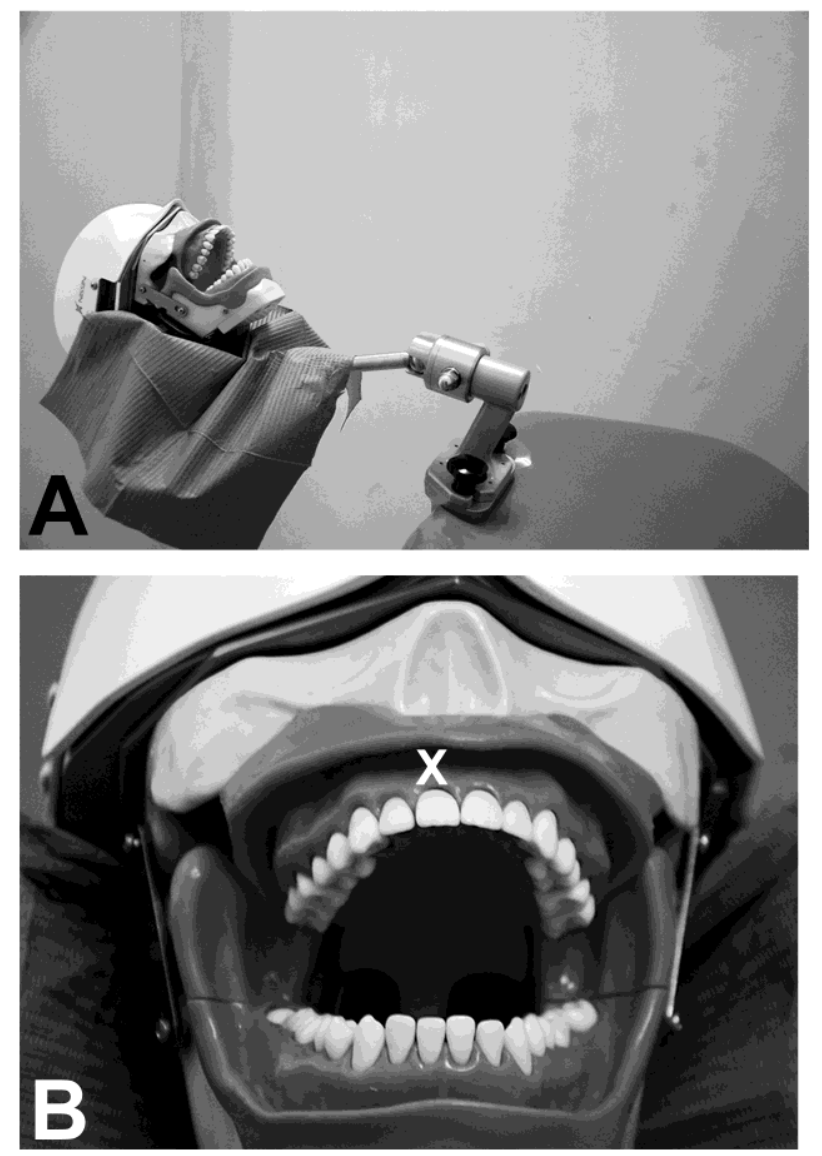

Fig. 1. The dental anaesthesia simulation model is adapted on the back of the dental chair (A). The puncture site is identified with an $\mathrm{X}(\mathrm{B})$.

duration of each operator's clinical practice was timed from the moment the anesthesia cartridge and dental needle were assembled on the dental syringe until the student recapped the dental needle. The average working time of each group was compared by Student's $t$-test to identify statistically significant differences.

\section{Evaluation of the students' perception of confidence}

The students' perception of confidence in performing the technique was evaluated based on 4 concepts: confidence in anatomical knowledge (CAN), confidence in performing the injection (CPI), confidence in managing the anesthesia technique (CMA), and confidence in controlling the hand for administering the anesthesia with a dental syringe $(\mathrm{CCH})$. These concepts were explored in an 8-item questionnaire answered by the students in G1 and G2 after performing the IASAN. The 4 concepts were paired with one item expressing a 
positive intention and one item expressing a negative intention. Thus, perception on CAN was expressed in items 1 and 5; perception on CPI was expressed in items 2 and 7; perception on CMA was expressed in items 3 and 6; and perception on $\mathrm{CCH}$ was expressed in items 4 and 8 . The items were quantified with a 5-point Likert scale. For positive items, the possible answers and values were: totally disagree $=1$, partially disagree $=2$, don't agree/don't disagree $=3$, partially agree $=4$, and totally agree $=5$. For negative items, the possible answers and values were: totally disagree $=5$, partially disagree $=4$, don't agree/don't disagree $=3$, partially agree $=2$, and totally agree $=1$. The average score of each item from G1 students was compared with that of G2 students, to identify possible statistically significant differences with a Mann-Whitney test. All levels of significance were set at $p<0.05$.

\section{RESULTS}

The success of the infiltrative technique for the administration of anesthesia was $100 \%$ for the 2 groups. The average working time was 9:36 $\mathrm{min} \pm$ 1:04 $\mathrm{min}$ and 15:12 $\mathrm{min} \pm$ 2:03 min for groups 1 and 2, respectively; the Student's $t$-test showed statistically significant differences $(p=0.03)$ between the results. Table 2 shows the average score obtained for each item of the questionnaire. Those in G1 showed higher average scores on the items exploring perception of confidence in performing the injection (items 2 and 7) as well as on the items exploring perception of the control of the hand (items 4 and 8). The average scores of these items showed statistically significant differences when compared between groups; however, no statistically significant differences were noted between the average scores for the items exploring the confidence in anatomical knowledge (items 1 and 5) and confidence in managing the anesthesia technique (items 3 and 6).

\section{DISCUSSION}

In this study, we evaluated dental students' perception when learning with the IASAN compared with a traditional technique. In addition, the IASAN working time was recorded for each participant, and the participants' success when using the IASAN was evaluated. Regarding the working time, students in G1 applied the IASAN in a shorter time than those in G2. A participant's first time performing the IASAN required skills in manipulating the 
Table 2. Post-clinical questionnaire and mean scores of group 1 and 2, with

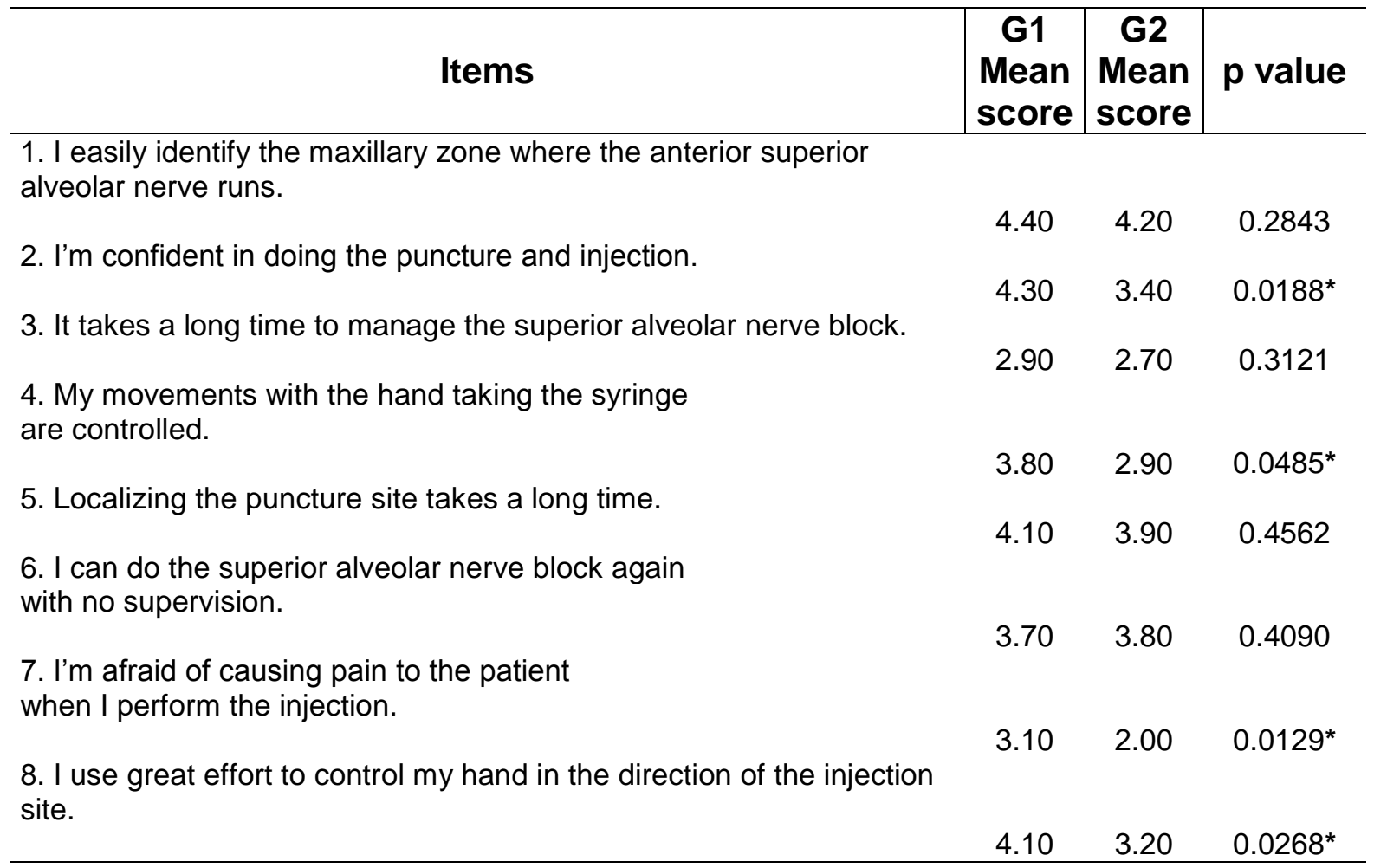

G1: group 1, G2: group 2. *:Statistically significant differences between mean scores.

\section{items quantified on a five-point Likert scale}

dental syringe (such as assembling the syringe with the dental cartridge and dental needle) and in handling the dental syringe for aspiration and injection. Hence, training with a simulation might improve the participants' technical-motor skills. A motor task might be completed in a shorter time by someone with higher skill, so a higher learning rate might correspond to a shorter time-scale (18). Other studies involving the administration of dental anesthesia did not investigate the impact of training on working time, nor did they evaluate whether the students with simulation-model training performed the anesthesia technique in a shorter time compared with the students without such training $(13,14)$. We measured the working time by recording time as a simple indicator (19), but more controlled methods should be explored in the future. The IASAN was 100\% successful for the 2 groups. In our study, success was evaluated based only on the absence of tooth sensitivity; this is distinct from success in pulpal anesthesia as measured in clinical situations (20). The success rate 
of the IASAN technique has been reported to be $>95 \%$ (16), and our results were similar, even though the students were performing the technique for the first time. The high success rate might be because IASAN is a technique with none of the anatomical or technical challenges faced in other techniques, such as inferior alveolar nerve blocking (IANB) (21).

Dental students' perception of their learning on the IASAN was investigated with a postintervention written test using a 5-point Likert scale; others have used a similar tool to evaluate the impact of simulation-based dental anesthesia learning $(9,13,14)$. The questionnaire was designed to explore 4 perceptions. The results showed that the students in G1 and G2 had similar perceptions of their anatomical knowledge (items 1 and 5). This perception concept involves theoretical knowledge that the participants reviewed several times; for instance, maxillary and anterior superior alveolar nerve anatomy was learned in a first-year course, and it was reviewed in stages 1 and 2. In addition, the puncture site at the apical third level of tooth 8 is easily located with visual exploration and manual palpation (16). Consequently, the 2 groups were easily able to identify the puncture site. For the perception of IASAN management (items 3 and 6), the 2 groups showed similar average scores. This was possibly-as already mentioned-because the students faced no anatomical or technical challenges in the application of the IASAN. The groups' perceived confidence levels in performing the injection were distinct: those in G1 showed a higher average score than those in G2 on items 2 and 7. Despite the fact that this is a common procedure, the administration of dental anesthesia is an action that can cause distress. Simon et al. reported that $18.8 \%$ of a group of dentists $(n=711)$ reported feeling distress when administering injections of anesthesia (22). Also, Chandrasekaran et al. observed anxiety in dental students performing a dental anesthesia technique for the first time (8). It is known, however, that practical courses and identification of equipment and techniques might increase confidence and decrease the risk of anxiety $(8,23)$, as we noted in our results. G1 students also showed a higher average perception of hand control (items 4 and 8). Thus, in this study, the simulation-based local anesthesia teaching strategy affected participants' perceptions of their technical skills. Motor-technical skills are gained in 3 stages: cognitive, integrative, and autonomous. In the cognitive stage, the student reflects on the theoretical bases of a procedure along with its steps and required technical skills (stages 1 and 2 of our training protocol); in the integrative stage, the student implements the procedure with 
tutorial support (stage 3 in our study: the simulation-based learning intervention); and in the autonomous stage, the student practices performing the procedure to learn skills (24). This evolving system occurred only for the G1 students, which might influence their perceptions of their technical skills. The preclinical use of a simulation model in local anesthesia teaching might support the 3-stage development of technical skills.

Other studies have evaluated simulation-based dental anesthesia interventions. Brand et al. observed that a simulation-based local anesthesia teaching strategy did not affect secondyear dental students' opinions after performing their first dental anesthesia injections; the simulation-trained students and simulation-untrained students showed similar perceptions regarding their experience in administering IANB, according to a post-intervention written test (with a 5-point Likert scale) (13). Our results disagree with those observations; in our study, we observed that training with the simulation affected the participants' perceptions. However, notable differences existed between our study and the other study; Brand et al. gave a theoretical lecture but did not describe the clinical demonstration or the simulationpreclinical training model. For instance, no information was given about the number of IANB repetitions or the duration of the training (13). However, those authors reported that the individuals receiving the IANB felt more comfortable receiving an injection from the students trained in administering anesthesia, a result ascribed to the use of the simulationtraining model (13). Another study that used a simulation-based dental anesthesia intervention, by Marei et al., evaluated the acquisition of theoretical and clinical knowledge by 3rd-year dental students who were trained or untrained with a DASM (14). One group of participants received instruction in a classroom with digital-visual media, and the other group received instruction in a simulation laboratory with electrical phantoms that activated a sound alarm when a dental needle targeted the correct puncture site. Four techniques were practiced, but neither the processes nor the number of repetitions was mentioned. The 2 groups completed a KAAT with a theoretical knowledge section and a clinical knowledge section, and no clinical injection was performed. The simulation-trained student showed a higher mean rank in both theoretical knowledge and clinical knowledge, but the differences were statistically significant only for the theoretical knowledge (14). A lack of clinical practice might influence the learning process, and if a simulation-based local anesthesia teaching strategy was applied, one would expect that learning would be reinforced. 
Simulation has been largely used in dental teaching, but little information exists about simulation for instruction on the administration of dental anesthesia; to date, it is unclear whether simulation is effective for students learning this subject. Effective protocols for dental anesthesia are needed to integrate and support the use of simulation systems in dental curricula. Our study overcame other studies' lack of information by listing the learning protocol and using repetitions of simulated injections in the model training, and the results showed that our simulation-based local anesthesia teaching strategy affected the perceptions of dental students. However, there were limitations. The study's small sample size might be questioned, since studies exploring simulation-based educational interventions have commonly involved a limited number of participants in trained and untrained groups, with a participant number of 8 through 13 being common $(19,25,26)$. Recently Lee et al. evaluated the effect of DASM on clinical learning by dental students. They included 30 participants in both the experimental and control groups, but even with that number of individuals, the DASM showed an unclear effect on the students' clinical learning (15). Another limitation of our study is that the impact of simulation on anesthesia success could be better tested with more complex techniques, since the IASAN is an easy technique relative to IANB and others used in simulation-model training. Our questionnaire could have used a larger item list to measure other perceptions, a point that should be considered for future studies.

\section{CONCLUSIONS}

Students trained with a simulation model for the administration of dental anesthesia showed higher values for perceived confidence in performing an injection and perceived hand control when compared with students without training. The DASM increased the value for perceived confidence in the performance of local dental anesthesia injections and for perceived handling of dental syringes. The students with training also performed the IASAN in a shorter time, although both groups had a $100 \%$ anesthesia success rate.

\section{ACKNOWLEDGMENTS}

The authors thank Zeyco S.A. de C.V for the donation of the anesthesia solution and topical anesthesia.

DISCLOSURE: The authors declare that they have no conflicts of interest. 


\section{REFERENCES}

1. Perry S, Bridges SM, Burrow MF. A review of the use of simulation in dental education. Simul Healthc. 2015;10:31-7.

2. Fugill M. Defining the purpose of phantom head. Eur J Dent Educ. 2013;17:e1-4.

3. Wolgin M, Wiedemann P, Frank W, Wrbas KT, Kielbassa AM. Development and evaluation of an endodontic simulation model for dental students. $J$ Dent Educ. 2015;79:1363-72.

4. Tubelo RA, Branco VL, Dahmer A, Samuel SM, Collares FM. The influence of a learning object with virtual simulation for dentistry: a randomized controlled trial. Int $J$ Med Inform. 2016;85:68-75.

5. Murray DJ. Progress in simulation education: developing an anesthesia curriculum. Curr Opin Anaesthesiol. 2014;27:610-5.

6. Udani AD, Kim TE, Howard SK, Mariano ER. Simulation in teaching regional anesthesia: current perspectives. Local Reg Anesth. 2015;8:33-43.

7. Rosenberg M. Simulation technology in anesthesiology. Anesth Prog. 2000;47:8-11.

8. Brand HS, Kuin D, Baart JA. A survey of local anaesthesia education in European dental schools. Eur J Dent Educ. 2008;12:85-8.

9. Chandrasekaran B, Cugati N, Kumaresan R. Dental students' perception and anxiety levels during their first local anesthetic injection. Malays J Med Sci. 2014;21:45-51.

10. Hossaini M. Teaching local anesthesia in dental schools: opinions about the studentto-student administration model. J Dent Educ. 2011;75:1263-9.

11. Brand HS, Tan LL, van der Spek SJ, Baart JA. European dental students' opinions on their local anaesthesia education. Eur J Dent Educ. 2011;15:47-52.

12. Said Yekta S, Lampert F, Kazemi S, Kazemi R, Brand HS, Baart JA, et al. Evaluation of new injection and cavity preparation model in local anesthesia teaching. $J$ Dent Educ. 2013;77:51-7.

13. Brand HS, Baart JA, Maas NE, Bachet I. Effect of a training model in local anesthesia teaching. J Dent Educ. 2010;74:876-9.

14. Marei HF, Al-Jandan BA. Simulation-based local anaesthesia teaching enhances learning outcomes. Eur J Dent Educ. 2013;17:e44-8. 
15. Lee JS, Graham R, Bassiur JP, Lichtenthal RM. Evaluation of a local anesthesia simulation model with dental students as novice clinicians. J Dent Educ. 2015;79:1411-7.

16. Malamed SF. Handbook of Local Anesthesia. St. Louis: Mosby, 2013: 188-191.

17. Cohen HP, Cha BY, Spangberg LS. Endodontic anesthesia in mandibular molars: a clinical study. J Endod. 1993;19:370-3.

18. Newell KM, Liu YT, Mayer-Kress G. Time scales, difficulty/skill duality, and the dynamics of motor learning. Adv Exp Med Biol. 2009;629:457-76.

19. Wulf G, Shea C, Lewthwaite R. Motor skill learning and performance: a review of influential factors. Med Educ. 2010;44:75-84.

20. Noguera-Gonzalez D, Cerda-Cristerna BI, Chavarria-Bolanos D, Flores-Reyes H, Pozos-Guillen A. Efficacy of preoperative ibuprofen on the success of inferior alveolar nerve block in patients with symptomatic irreversible pulpitis: a randomized clinical trial. Int Endod J. 2013;46:1056-62.

21. Hargreaves KM, Keiser K. Local anesthetic failure in endodontics. Endod Topics. 2002;1:26-39.

22. Simon JF, Peltier B, Chambers D, Dower J. Dentists troubled by the administration of anesthetic injections: long-term stresses and effects. Quintessence Int. 1994;25:641-6.

23. Meechan JG. Differences between men and women regarding attitudes toward dental local anesthesia among junior students at a United Kingdom dental school. Anesth Prog. 2005;52:50-5.

24. Lachapelle K. Teaching technical skills using medical simulation: a new frontier. McGill J Med. 2007;10:149-51.

25. Buchanan JA. Experience with virtual reality-based technology in teaching restorative dental procedures. J Dent Educ. 2004;68:1258-65.

26. Gottlieb R, Lanning SK, Gunsolley JC, Buchanan JA. Faculty impressions of dental students' performance with and without virtual reality simulation. J Dent Educ. 2011;75: 1443-51. 